\title{
An Enhanced R-NSGA-II For Multiple Brands Advertising Campaign Allocation Problem
}

\author{
Fodil Benali, Damien Bodénès \\ Adwanted Group \\ Paris, France \\ \{fbenali,dbodenes\}@adwanted.com
}

\author{
Cyril De Runz, Nicolas Labroche \\ BDTLN - LIFAT, University of Tours \\ Blois, France \\ \{cyril.derunz, nicolas.labroche\}@univ-tours.fr
}

\begin{abstract}
This paper deals with the Campaign Allocation Problem of commercial Ads in TV breaks that we formalize as a multi-stakeholders multiobjective problem with highly competing objectives for different brands and numerous constraints. The problem is NP-hard with a high dimensional objective space and scalability issues in terms of the number of breaks. Moreover, the expected solution should be able to focus on a sub-part of the Pareto front according to decision maker's (DM) knowledge. To tackle these challenges, we propose to use R-NSGA-II, a ManyObjective Evolutionary Algorithm (MaOEA), combined with a novel gene encoding/decoding process. Experiments show that this approach obtains better results than usual MaOEA (NSGAII, NSGA-III) according to industrial performance criteria, scales to large instances, and incorporates decision maker's preferences during the optimization process.
\end{abstract}

Index Terms-Advertising, scheduling problems, evolutionary algorithms, many-objective optimization

\section{INTRODUCTION}

The TV advertisements scheduling problem is defined as follows: given a set of advertisers' campaigns and a set of commercial breaks with air times (slots), the Campaign Allocation Problem (CAP) [1] is to determine how campaigns' spots (brands messages) should be allocated to a subset of breaks in order to maximize the total revenue of the TV networks and evenness with respect to the advertisers' requirements and limited advertising inventory restrictions. It is an NP-hard combinatorial multiobjective optimization problem that involves conflicting objectives and complicated constraints [2]. One approach to solve this problem is to consider 2 sub-problems: (1) determining the best assignment of concurrent brands' spots to available breaks, (2) inside each break, optimizing the sequence of spots, by taking into account the importance of the first and last slots of a break.

This paper deals with Problem (1) for which there already exists an extensive literature, noticeably in the operation research community such as [3]. These approaches tend to formulate the problem based on a single objective with a large number of constraints and in the case of multi-campaigns, they treat the campaigns either sequentially or by considering an aggregated objective. Another approach [4] considers the use of a multi-objective evolutionary algorithm by searching to optimize one objective per campaign (audience coverage) but it

This work is funded by the ANRT CIFRE Program (2019/0877). does not consider the other objectives to reach for a campaign nor the TV networks objectives for instance.

Contrary to previous works, in our context, we consider several stakeholders: advertisers, and TV networks, each with its own objectives. We also consider the size of the problem instance, all breaks from several months, that may not be appropriate for an exact resolution based on solvers. Therefore, there is a need for a multi-objective optimization algorithm that would provide the Decision Maker (DM), i.e. planner, with a set of Pareto-optimal solutions to decide which one should be used as the best solution. Evolutionary algorithms (EAs) [5] is well-suited to solve multi-objective problems [6] thanks to their ability to provide a set of trade-off solutions in addition to their insensitivity to the geometrical features of the objective space. However, in our context, using an EA still raises challenges:

- Many-objective problem: as we consider a large number of objectives, most solutions become equivalent to each other. Therefore, finding solutions that are better compared to others according to the whole set of objectives is really challenging and requires adapted techniques to support the optimization.

- Constrained problem: our EA should search for solutions in the decision space while respecting a set of predefined constraints so that it outputs a set of feasible non-dominated solutions.

- Semi-supervised context: we propose to take advantage of any a priori preferences on the objectives. In this paper, we focus on a specific subset of the Pareto-optimal set according to a reference point that drives the convergence of the EA.

To address the aforementioned challenges, this paper: (i) proposes a formalization of this optimization problem to be solved by an evolutionary algorithm, (ii) introduces a preliminary encoding / decoding of solutions, represented as assignments of spots to breaks, that manages some of our constraints and can scale to large problem instances, and finally (iii) describes experiments that validate our formalization, the use of a reference based evolutionary algorithm, and the solution scalability. 


\section{PROBlEM DESCRIPTION AND FORMALIZATION}

Scheduling TV advertisements involves two types of participants that are television networks (channels) and advertisers. After announcing TV shows programs, the TV networks finalize their rating forecasts and set the rate cards for the available advertising breaks. The rate cards contain for each break:

- The gross price for 1-second of message broadcasting.

- Whether it is a Prime break, i.e. an audience peak period.

- Expected gross rating point (GRP), which estimates the percentage of the audience during the commercial break.

- The date and the timing of the break.

Once rate cards are ready, the commercial break slots are exposed for selling. Then, the advertisers buy the slots in order to obtain the most efficient ad campaign. An advertiser's request corresponds to one brand's advertising campaign containing:

- The budget to invest for one brand's ad campaign.

- The cost per one GRP (CPP).

- Percentage of budget to invest in Prime breaks.

- The advertising spot duration (e.g., 15 seconds).

- The brand's competition code, which allows to avoid slot allocation of competitive brands in the same break.

The advertising campaign optimization consists in obtaining a distribution of the available commercial break slots that maximize the invested budget allocation and achieves the best GRP for each brand while maximizing the revenue of the TV networks and maintaining the clients' loyalty. Moreover, for each campaign, there is a rate of Prime breaks to reach. This leads to a multi-stakeholders many-objective setting with competing objectives for different brands and various constraints.

The obtained distribution must take into account clients' constraints and TV networks' inventory restrictions. The major clients' requests constraints are the maximum budget allowed to spend, and the list of brands' commercials that are allowed to be exposed in the same break based on competitive exclusion rules. Besides, the sum of advertised spot duration cannot exceed the break length capacity.

In order to formally present the problem, the following notation is used.

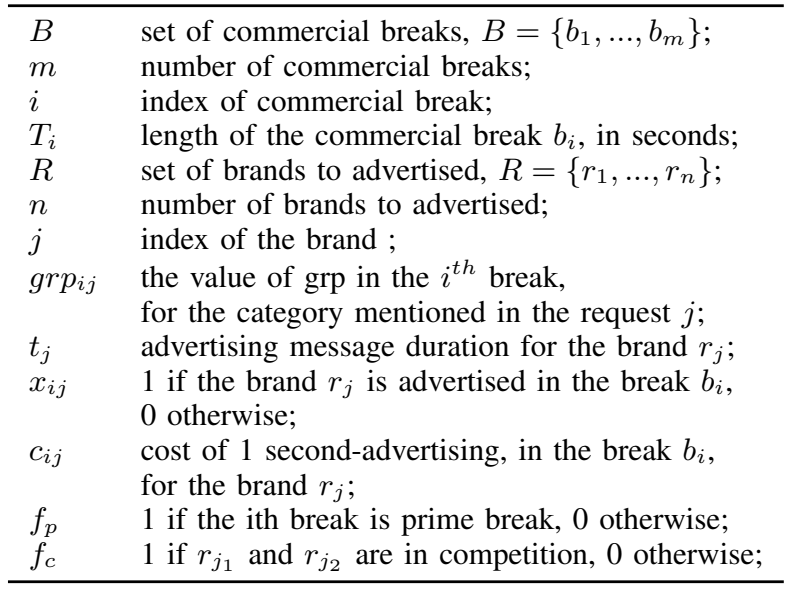

The mathematical problem can be formulate as follows:

$$
\begin{gathered}
\min \left|\sum_{i=1}^{m} x_{i j} * g r p_{i j}-G R P_{j}\right|, j \in \llbracket 1 ; n \rrbracket \\
\min \left|\sum_{i=1}^{m} x_{i j} * c_{i j} * t_{j} * f_{p}(i)-P R I M E_{j}\right|, j \in \llbracket 1 ; n \rrbracket \\
\max \sum_{i=1}^{m} \sum_{j=1}^{n} x_{i j} * c_{i j} * t_{j} \\
\max \sum_{i=1}^{m} \sum_{j=1}^{n} x_{i j} * P R I O R I T Y_{j}
\end{gathered}
$$

Subject To:

$$
\begin{gathered}
\sum_{i=1}^{m} x_{i j} * c_{i j} * t_{j} \leq B U D G E T_{j}, j \in \llbracket 1 ; n \rrbracket \\
\sum_{j=1}^{n} x_{i j} * t_{j} \leq T_{i}, i \in \llbracket 1 ; m \rrbracket
\end{gathered}
$$

$\sum_{i=1}^{m} \sum_{j_{1}=1}^{n} \sum_{j_{2}=1}^{n}\left(x_{i j_{1}} * x_{i j_{2}} * f_{c}\left(j_{1}, j_{2}\right)\right)=0, \quad j_{1} \neq j_{2}$

1) Client Side: Optimize for each client's brand $r_{j}$, a set of objectives defined as follows:

- Objective function (1) consists in maximizing the GRP goal attainment, by minimizing the absolute difference between the achieved GRP and the aimed GRP $\left(G R P_{j}\right)$.

- Objective function (2) maximizes budget invested in prime breaks, by minimizing the absolute difference between the allocated prime breaks budget and the invested prime breaks budget $\left(P R I M E_{j}\right)$.

In the context of media planning, we have to maximize the budget allocation for the clients' requirement satisfaction, without exceeding the maximal budget allowed of each client. Hence, a budget constraint (5) is needed for each brand.

2) TV Networks Side: a global optimization is required:

- Objective function (3), maximizes the total revenue of the TV Networks.

- Objective function (4), maximizes slots allocation based on client's potential and loyalty (PRIORITY $\left.Y_{j}\right)$.

Finally, the solutions must respect the Commercial break length constraint (6) and not allowing competing brands to be advertised in the same break (7).

\section{Many-OBJective Evolutionary Algorithms}

There are two main approaches to deal with Multi-Objective Problems (MOP) with EAs. First, approaches that consider each objective individually as in Vector Evaluated Genetic Algorithm (VEGA) [7], , or the well-known NSGA-III [8], However, they are sub-optimal as VEGA does not promote the survival of good trade-off solutions while NSGA-III relies on multiple parameters.

Second, evolutionary methods that deal with all objectives at the same time. Among them, the Non-dominated Sorting Genetic Algorithm (NSGA-II) [9] is the most well-known. NSGA-II is an elitist algorithm that uses two types of fitness functions. Although NSGA-II has demonstrated to be one of 
the most competitive MOEAs [5] as it is simple, parameterless, and has a low computational complexity of $\mathrm{O}\left(M N^{2}\right)$ ) with elitist approach. However, it faces difficulties when dealing with a large objective number [10] due to dimensionality.

Nevertheless, recent studies [11] have shown that NSGAII can be improved in many-objective contexts by focusing on a smaller subset of the Pareto optimal solutions close to a supplied set of reference points. For instance, in the Reference point-based NSGA-II algorithm (R-NSGA-II) [12], a set of DM's specified reference points can be used in a semisupervised way to guide the search in the objective space and to control the diversity of the focused Pareto-set.

\section{PRoposed Solution}

\section{A. Solution Encoding}

The most challenging task in campaign allocation optimization for multiple brands requests is the effective constraints handling in a high dimensional search space context. As a consequence, our solution proposes an encoding phase encompassing the handling of hard and computational consuming constraints such as commercial break length constraint (6) and brands competition constraint (7) before the search space exploration. For this reason, the encoding process is done in three steps:

1) Break allocation combinations generation: for each break $b_{i}$, we generate all possible break allocation combinations. Then, we filter solutions that violate the constraints.

2) Break allocation combinations sorting based on the rate of completeness: for each break combination, we calculate its rate of completeness by dividing the sum of allocated spots in the combination by the break length. After that, the combinations are sorted based on their rate of completeness.

3) Break allocation combinations encompassing into state based representations: for each break $b_{i}$, we map the list of combinations to a list of states, where each state is defined as the index of the combination.

To include the previous encoding in R-NSGA-II algorithm, the following notation is used:

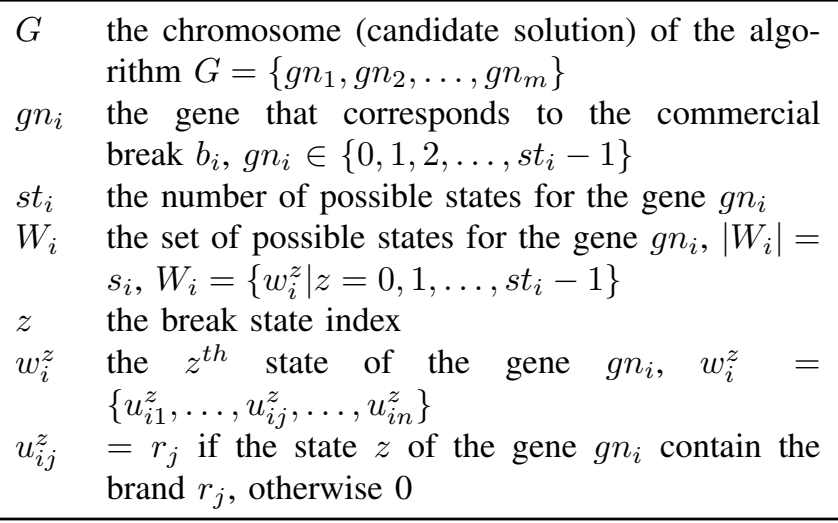

This implies that advertisements for the break $b_{i}$ are coded in the gene $g n_{i}$ by a break state index $z$. There is a bijective mapping between the break-state and actual break spots.

The major advantage of this encoding is the search space dimensionality reduction. In fact, the usual representation of chromosomes represents each solution vector by $m * n$ variables, which becomes rapidly intractable: for $m=100 \mathrm{k}$ breaks, and $n=10$ brands requests, solution vectors of 1 million variables are required which conducts us to the curse of dimensionality and search space sparsity. However, using our representation, we reduce the search space dimensionality to $m$ variables only. Moreover, since our representation does not depend on the clients' requests, obtained solutions could be used as an initial population for future brand request handling.

\section{B. Population Initialization}

To generate the initial population, we propose to initialize the population with individuals of empty break states. The idea behind is that the genetic algorithm principle consists in building optimal solutions by assembling small building blocks. As building blocks are brought together, we get closer to this optimal solution. Consequently, initializing the population with empty breaks will allow the heuristic to progress incrementally towards the optimal solution.

\section{Crossover and Mutation}

We perform the mutation of the solution $G=$ $\left\{g n_{1}, g n_{2}, \ldots, g n_{m}\right\}$ on state-wise basis, with all genes having an equiprobability $p_{m}\left(p_{m}=\frac{1}{m}\right)$. If the gene $g n_{i}$ is selected for the mutation, we suggest two options:

- The first option consists in randomly changing its value to one of the equiprobable states $\left\{0,1,2, \ldots, s t_{i}\right\}$

- The second option considers a mutation mechanism which favors the states which fill or maximize the completeness of the breaks in the beginning and have small broadcasts at the end.

One of the most challenging tasks in CAP is the TV networks' revenue management. In fact, optimizing revenue requires minimizing losses resulting from small residual durations in TV breaks due to partial reservation: if we assign a 25second spot to a 30-second TV break, the residual 5 seconds will be difficult to sell. As a consequence, our technique will allow to control the breaks allocations completeness rate and reduce the number of breaks partially reserved.

We use a single-point crossover consisting in picking randomly a crossover point after that the two parent solutions are swapped before and after the single point. The advantage of this step is to exploit the search space between the two given solutions.

\section{Solution Decoding}

To evaluate candidate solutions, chromosomes need to be decoded. For that, we use the sets of mapping from the encoding step. Since the sets of the possible states $W_{i}$ are computed prior to the EA starts, having a set of such mappings for each break enables fast coding and decoding of the solutions during the algorithm run. 


\section{E. Evaluation and Selection}

Once the population of solutions is decoded, for each nonevaluated-yet solution, we evaluate:

- For each brand request, the GRP attainment objective (1), and the prime budget objective (2).

- The revenue optimization objective (3), and the priority objective (4) for the TV networks.

After the solution evaluation, the selection process is done in 4 steps:

- Infeasible solutions, i.e., solutions that violate the budget constraint (5), are removed from the population.

- A non-dominated sorting is performed to classify the population into different levels of non-domination.

- Solutions from the best non-domination levels are chosen front-wise.

- For solutions from the last front which cannot be entirely chosen to maintain the population size of the next population, the preference based distance [12] is used to select a subset of diverse solutions based on the reference point selected by the DM.

\section{COMPUtational Experiments}

In this section, we report the experiments that were conducted to show the applicability and validity of the proposed approach and mathematical modeling to solve the campaign allocation problem.

In order to evaluate our approach, we answer the following research questions:

1) How does our model R-NSGA-II deal with many objectives, and how beneficial is the reference point in our context?

2) To which extent the proposed encoding of solutions is efficient for handling constraints in high dimensional search space?

\section{A. Experimentation Design}

We generate 2 synthetic datasets based on confidential French advertising data and using a the synthetic data generation framework based on copula called, MTCopula [13]. The first dataset contains 1000 breaks ( $m=1000$, i.e., low DSS) and the second dataset contains 10000 breaks ( $m=10000$, i.e., high DSS). The experiment parameter configuration is set as follows: the population size is set to 40 , the time budget (stopping criterion) is set to $180 \mathrm{sec}$. Together, these two parameters imply a fair and objective evaluation of the algorithms. EA operators parameters are set to standard configurations: the mutation probability $p_{m}=1 / \mathrm{m}$ and the crossover probability $p_{c}=1$. To assess the performance, we evaluate the objective functions using 6 randomly generated client requests. Finally, due to the stochastic nature of evolutionary algorithms, each experiment has been repeated 20 times. As the obtained results are comparable, we only report one result for each experiment for the sake of readability.

\section{B. R-NSGA-II Vs NSGA-II and NSGA-III}

To validate the choice of R-NSGA-II algorithm in our approach, we study the performance of the proposed solution pipeline using three algorithms R-NSGA-II, NSGA-II, and NSGA-III. Each time, we evaluate the absolute percentage error of the GRP attainment (1) and the Prime budget achieved (2) for the 6 brand request competing over a pool of 1000 commercial breaks $(m=1000)$. Noticeably, reference directions in NSGA-III have been generated using Das-Dennis method. The reference point in R-NSGA-II is defined by the optimal value for each objective function.

Fig. 1 presents the evaluation of clients' criterion optimization based on the percentage absolute error. We observe clearly that clients' requirements (GRP and Prime budget) are better respected using the R-NSGA-II with a small variation error that does not exceed 0.05 for the GRP attainment and 0.14 for the Prime budget, when they are large GRP and Prime errors for both NSGA-II and NSGA-III with a variation that exceeds $50 \%$ in the case of NSGA-III. This is because NSGA-II behaves like a random search in high DSS, and NSGA-III requires many parameters setting (population size and reference directions) to be set cautiously on a case-by-case basis.

\section{Interest Of Encoding Process}

In order to show the utility of the proposed encoding process for handling hard constraints such as break length constraints (6) and competing constraint (7), we evaluate our approach by activating and deactivating the process of encoding. Each time, the absolute error of the clients objective functions (1)(2) is used to assess efficiency of this process. We have to mention here that the deactivation of the process implies checking the constraints during the optimization process and high DSS to represent solutions ( $\left.n^{*} \mathrm{~m}\right)$. Fig. 2 shows the impact of activating and deactivating the process of encoding on the quality of the optimization. Deactivating the process of encoding decreases the overall performance of the approach because, due to the high number of constraints, a lot of otherwise rejected solutions are kept, and as evolutionary heuristics build solutions incrementally based on last identified feasible solutions. As a consequence, we notice a variation of errors between 0.60 and 1.0 for both of the GRP and Prime budget for all client requests. This shows that this encoding is efficient to simultaneously handle efficiently multiple constraints.

\section{CONCLUSION}

In this paper, we proposed a new optimization approach for concurrent brand spots assignment to available breaks in TV networks. This industrial goal led us to first propose a multistakeholders many-objective formalization of the problem with numerous constraints and highly competing brand objectives. In order to tackle this latter, we proposed an enhanced RNSGA-II which incorporates DM's preferences and uses custom operators with an efficient problem encoding. Several experiments validate the interest of the proposal. 


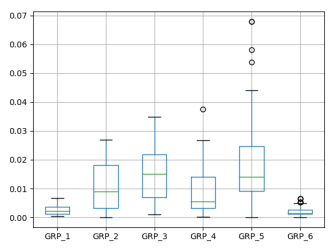

(a) GRP Error Using R-NSGA-II

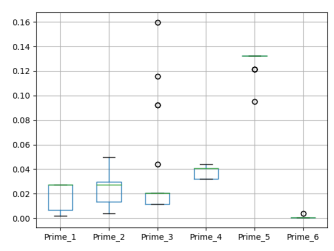

(d) Prime Error Using R-NSGA-II

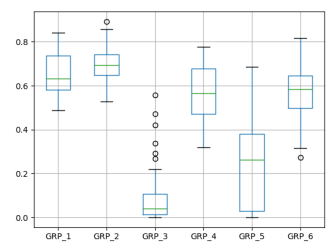

(b) GRP Error Using NSGA-II

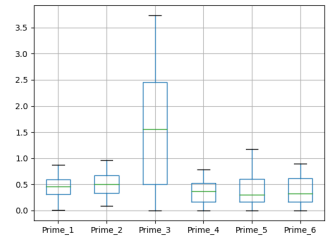

(e) Prime Error Using NSGA-II

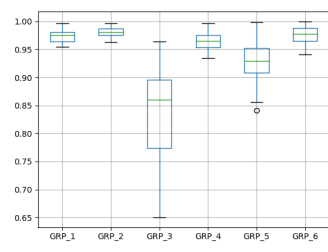

(c) GRP Error Using NSGA-III

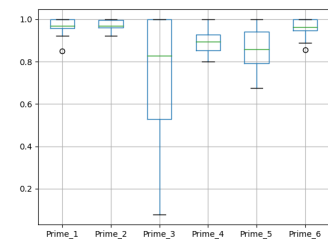

(f) Prime Error Using NSGA-III

Fig. 1. Absolute percentage error evaluation for the GRP and Prime Budget of 6 requests using R-NSGA-II, NSGA-II, and NSGA-III.

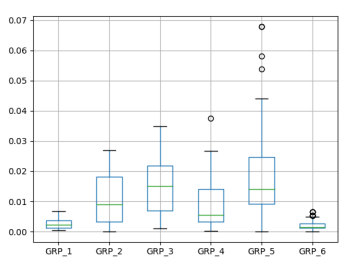

(a) GRP error using encoding

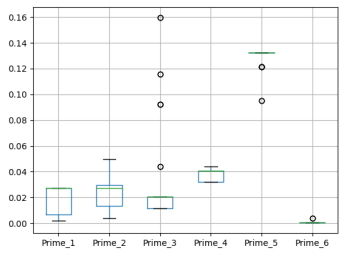

(c) Prime error using encoding

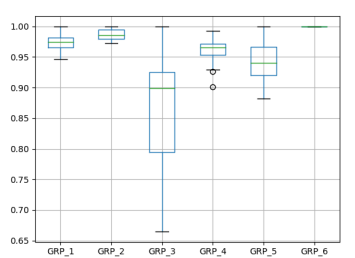

(b) GRP error without encoding

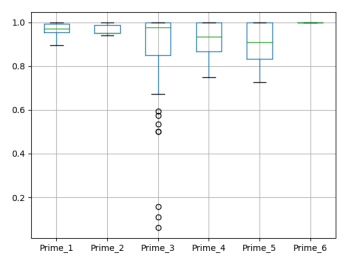

(d) Prime error without encoding
Fig. 2. Impact of activating and deactivating the encoding process.

\section{REFERENCES}

[1] A. Mihiotis and I. Tsakiris, "A mathematical programming study of advertising allocation problem," Applied Mathematics and Computation, vol. 148, no. 2, pp. 373379, 2004.

[2] T. Benoist, E. Bourreau, and B. Rottembourg, "The TVbreak packing problem," European Journal of Operational Research, vol. 176, no. 3, pp. 1371-1386, 2007.

[3] X. Zhang, "Mathematical models for the television advertising allocation problem," International Journal of Operational Research, vol. 1, no. 3, pp. 302-322, 2006.

[4] P. J. Fleming and M. A. Pashkevich, "Optimal advertising campaign generation for multiple brands using MOGA," IEEE trans. on Systems, Man, and Cybernetics, Part C, vol. 37, no. 6, pp. 1190-1201, 2007.
[5] S. Chand and M. Wagner, "Evolutionary many-objective optimization: A quick-start guide," Surveys in Operations Research and Management Science, vol. 20, no. 2, pp. 35-42, 2015.

[6] S. Ghambari, M. Golabi, J. Lepagnot, M. Brévilliers, L. Jourdan, and L. Idoumghar, "An Enhanced NSGA-II for Multiobjective UAV Path Planning in Urban Environments," in ICTAI. IEEE, 2020, pp. 106-111.

[7] J. D. Schaffer, "Multiple objective optimization with vector evaluated genetic algorithms," in Proceedings of the first international conference on genetic algorithms and their applications, 1985. Lawrence Erlbaum Associates. Inc., Publishers, 1985.

[8] K. Deb and H. Jain, "An evolutionary many-objective optimization algorithm using reference-point-based nondominated sorting approach, part i: solving problems with box constraints," IEEE trans. on evo. computation, vol. 18, no. 4, pp. 577-601, 2014.

[9] K. Deb, A. Pratap, S. Agarwal, and T. Meyarivan, "A fast and elitist multiobjective genetic algorithm: NSGAII," IEEE trans. on evo. computation, vol. 6, no. 2, pp. 182-197, 2002.

[10] H. Ishibuchi, N. Tsukamoto, Y. Hitotsuyanagi, and Y. Nojima, "Effectiveness of scalability improvement attempts on the performance of NSGA-II for many-objective problems," in GECCO'08, 2008, pp. 649-656.

[11] H. Ishibuchi, T. Matsumoto, N. Masuyama, and Y. Nojima, "Many-objective problems are not always difficult for pareto dominance-based evolutionary algorithms," in ECAI 2020. IOS Press, 2020, pp. 291-298.

[12] K. Deb and J. Sundar, "Reference point based multiobjective optimization using evolutionary algorithms," in GECCO’06, 2006, pp. 635-642.

[13] F. Benali, D. Bodénès, N. Labroche, and C. de Runz, "MTCopula: Synthetic Complex Data Generation Using Copula," in DOLAP, 2021, pp. 51-60. 